\title{
The non-invasive recognition of left atrial enlargement: comparison of electro- and echocardiographic measurements
}

\author{
HAMID IKRAM \\ M.D., F.R.C.P.E., M.R.C.P., M.R.A.C.P. \\ P. J. BONES \\ W. Chan \\ M.B., B.S. \\ Department of Cardiology, The Princess Margaret Hospital, \\ Christchurch, New Zealand
}

\begin{abstract}
Summary
The aim of this study was to compare the ability of electro- and echocardiography to detect enlargement of the left atrium. Seventy-four patients, divided into three groups (eighteen normal, thirty-six valvular disease, twenty hypertension and/or coronary artery disease) were studied. The $P$ wave terminal force in lead $V_{1}\left(P T F-V_{1}\right)$ was measured from a standard 12 lead electrocardiogram, and the internal left atrial dimension (LAD) was measured from time-motion echocardiograms. Linear regression analysis showed a small but significant linear correlation between PTF-V ${ }_{1}$ and LAD $(r=0.32, P<0.01)$. Both methods would separate patients with diseases known to cause left atrial enlargement from normals, but echocardiography showed greater 'specificity' $(100 \%$ v. 94\%) and 'sensitivity' $(75 \%$ v. $67 \%)$. It was much superior to the ECG in detecting milder grades of left atrial enlargement and for following serial changes.
\end{abstract}

\section{Introduction}

The detection of left atrial enlargement or its progression is frequently important in clinical medicine. The techniques traditionally used to evaluate left atrial size have been X-ray examination of the barium-filled oesophagus, angiocardiography and electrocardiography.

All these techniques have drawbacks. Angiography requires cardiac catheterization and cannot be regarded as a routine test. Barium studies entail a great deal of co-operation from the patient, require extensive radiological facilities and, because of the radiation hazard, they cannot be performed on pregnant women or be frequently repeated. Also barium studies can detect only severe grades of left atrial enlargement: with lesser grades of severity and

Correspondence: Dr H. Ikram, Department of Cardiology, The Princess Margaret Hospital, Cashmere Road, Christchurch 2, New Zealand. with concomitant left ventricular enlargement, falsenegatives are frequent.

Hence there is need for a simple, non-injurious and frequently repeatable technique for reliably assessing left atrial size.

The choice currently lies between the electrocardiogram and the echocardiogram.

The electrocardiogram is free from the traumatic, radiation and economic drawbacks of angiographic and barium studies. Many criteria have been proposed for the cardiographic recognition of left atriak enlargement all based on the $\mathbf{P}$ wave. They include the classical ' $P$ mitrale' (Lewis, 1920; Berliner and Master, 1938), the ratio of the duration of the $P$ wave to the PR segment also called the Macruz Index (Macruz, Perloff and Case, 1958) and the product of the P terminal force duration (in sec) and its depth (in $\mathrm{mm}$ ) in lead $\mathrm{V}_{\mathbf{1}}$. The latter is generally regarded as the best electrocardiographic criterion for detecting left atrial enlargement (Morris et al., 1964; Hurst et al., 1963; Soloff and Zatuchini, 1958).

The electrocardiographic diagnosis of left atrial enlargement suffers from a variety of limitations. These include the technical problems of recording and accurately measuring very small deflections, the inability to distinguish between 'pressure overload' and true anatomical enlargement (Pipberger and Tanenbaum, 1958; Kasser and Kennedy, 1969). The loss of the $\mathbf{P}$ wave from atrial fibrillation makes ECG detection of left atrial enlargement impossible.

Despite these limitations the ECG is widely used to assess left atrial size in clinical medicine (Arevalo, Spagnuolo and Feinstein, 1963; Ishikawa, Kini and Pipberger, 1973; Gooch et al., 1966; Human and Snyman, 1963).

The recent technique of echocardiography also enables left atrial size to be measured (Feigenbaum, 1972; Hirata et al., 1969). Since this is a non-invasive, non-radiological technique, it is comparable to the 
ECG in its safety and ease of performance. In contrast to the ECG, left atrial dimension of the echocardiogram is uninfluenced by acute rise in left atrial pressure (Chandraratna, Nanda and Shah, 1976).

Whilst echo measurements of left atrial size have been shown to correlate well with left atrial volumes determined by angiography (Hirata et al., 1969; Ten Cate et al., 1974), there is little information on how reliable each technique is in detecting left atrial enlargement in the purely clinical situation (Termini and Yu-Chen Lee, 1975; Chirife, Feitosa and Franki, 1975).

The purpose of this communication is to report a comparison of the $P$ terminal force in lead $V_{1}$ as the ECG index of left atrial size with echocardiographic measurements of the same chamber in the diagnosis of clinical left atrial enlargement.

\section{Materials and methods}

The material consisted of three groups based on presumed left atrial enlargement.

\section{Group 1}

Normal subjects with no history or clinical evidence of heart disease and thus normal sized left atria (eighteen cases).

\section{Group 2}

Patients with chronic aortic (eighteen) and mitral valve disease (eighteen) and enlarged left atria (thirty-six cases).

\section{Group 3}

Hypertensive ischaemic heart disease. They may have mild, moderate or no left atrial enlargement (twenty cases).

The $P$ wave negative terminal force in lead $V_{1}$ $($ PTF-V 1 ) was measured using an illuminated magnifier with $0.1 \mathrm{~mm}$ graduated scale from a standard 12 lead electrocardiogram. The depth of the negative deflection of the $\mathbf{P}$ wave was measured from the isoelectric line to the point where the greatest deflection occurred. The duration of the negative deflection was measured between the points of intersection of the leading edge of the trace with the isoelectric line (Fig. 1). The deflection and duration were multiplied together to give PTF-V $\mathrm{V}_{1}$ (1 unit = $0.00004 \mathrm{mV} / \mathrm{sec}$ ).

The echocardiograms were recorded with a Smith Kline Ekoline 20A ultrasonoscope with a $2.25 \mathrm{MHz}$ transducer ( $5 \mathrm{~cm}$ focal length). The patients were studied in a semi-recumbent left lateral position with the transducer position over the third or fourth intercostal space near the left sternal border. The anterior leaflet of the mitral valve was identified and the transducer was rotated medially and slightly

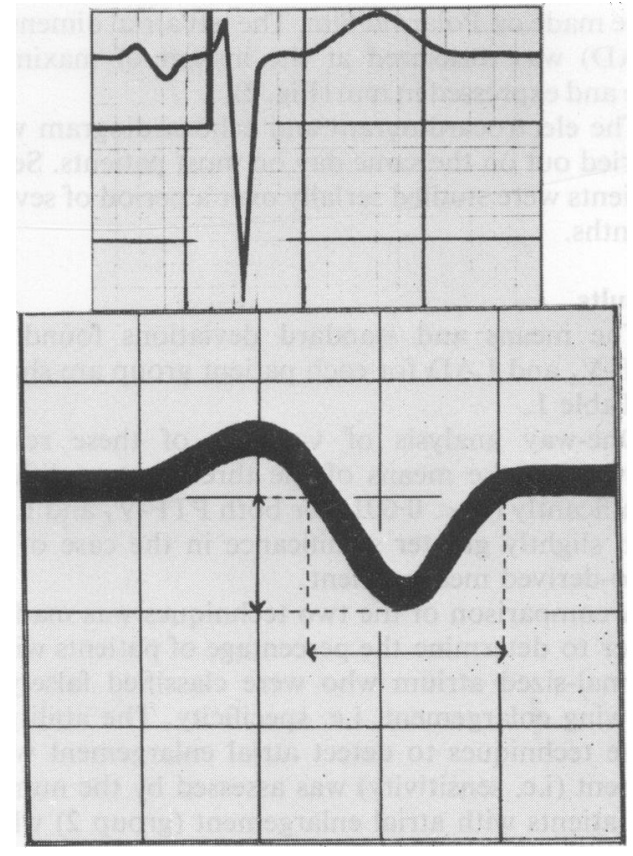

FIG. 1. Measurement of $P$ terminal force in lead $V_{1}$ (PTF-V 1 ).

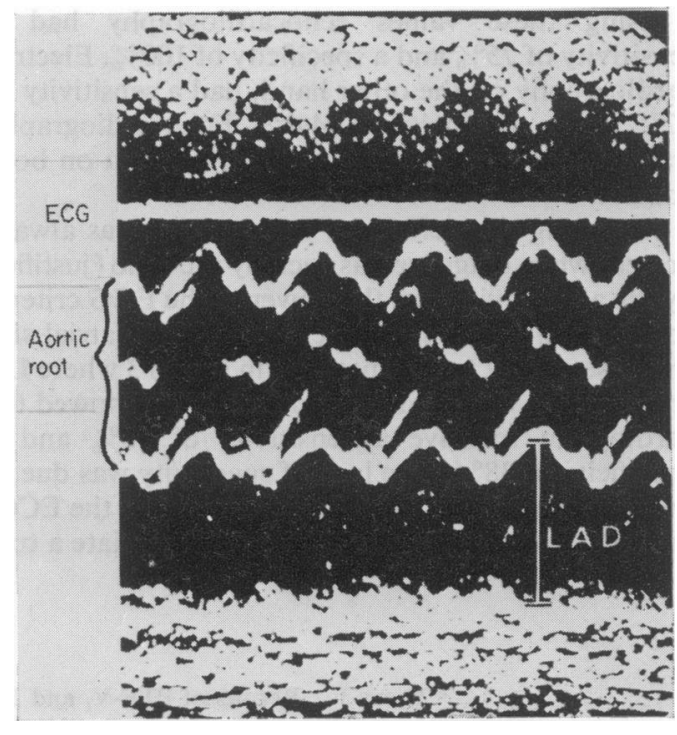

Fig. 2. Measurement of left atrial dimension (LAD).

superiorly to obtain the aortic root and left atrial echocardiogram. A time-motion (M mode) display was produced on the Ekoline oscilloscope, including a bipolar electrocardiogram, and permanent records 
were made on Polaroid film. The left atrial dimension (LAD) was measured at the instant of maximum size and expressed in $\mathrm{mm}$ (Fig. 2).

The electrocardiogram and echocardiogram were carried out on the same day on most patients. Seven patients were studied serially over a period of several months.

\section{Results}

The means and standard deviations found for PTF-V $V_{1}$ and LAD for each patient group are shown in Table 1.

One-way analysis of variance of these results shows that the means of the three groups differed significantly $(P<0.001)$ for both PTF-V ${ }_{1}$ and LAD with slightly greater significance in the case of the echo-derived measurement.

A comparison of the two techniques was made in order to determine the percentage of patients with a normal-sized atrium who were classified falsely as showing enlargement, i.e. specificity. The ability of these techniques to detect atrial enlargement when present (i.e. sensitivity) was assessed by the number of patients with atrial enlargement (group 2) which were classified as such.

Upper limits of normal were defined as the mean value plus $1.65 \mathrm{~s} . d$. (single tailed $95 \%$ level) and were 27.7 units and $29 \mathrm{~mm}$ for PTF- $\mathrm{V}_{1}$ and left atrial dimension respectively.

Using these values echocardiography had a sensitivity of $75 \%$ and a specificity of $100 \%$. Electrocardiography on the other hand, had a sensitivity of $67 \%$ and a specificity of $94.4 \%$. Echocardiography was thus superior to the electrocardiogram on both counts.

Assuming that left atrial enlargement was always present when diagnosed as such by the echo (justified by the above evidence) the power of the ECG criteria correctly to detect smaller changes in left atrial size was assessed by testing patients in group 3 where LA enlargement was mild to moderate. This showed the cardiogram to have a sensitivity of $58 \%$ and a specificity of $38 \%$. The lack of specificity was due to over diagnosis of left atrial enlargement by the ECG, probably owing to its inability to differentiate a true increase in left atrial size from elevation in left atrial pressure.

On twenty-six patients from group 2 and group 3, the aortic root dimension (Ao) was also measured from echocardiograms. The left atrial dimension/ aortic root ratio has been suggested as a more accurate predictor of left atrial enlargement than left atrial dimension (Ten Cate et al., 1974). The results of this study, however, showed no significant difference between the two methods.

Serial studies in seven patients with left ventricular failure demonstrated a clear decreasing or increasing trend in left atrial dimension consistent with the improvement or worsening of the patients' clinical condition. No such relationship was evident in the PTF- $V_{1}$ results on the same patients, quite large variations occurring in some cases.

\section{Discussion}

This study indicates that echocardiography was better than the electrocardiogram in detecting LA enlargement in the presence of valvular heart disease. This may be because the terminal portion of the $P$ wave is influenced by factors other than left atrial size. Thus simultaneous enlargement of other chambers, abnormal thoracic anatomy and changes in left atrial pressure without enlargement are known to produce $P$ wave changes indicative of enlargement (Saunders et al., 1967; Romhilt et al., 1972; Morris et al., 1966). Furthermore, the onset of atrial fibrillation, a common complication in most conditions associated with left atrial enlargement, precludes the use of criteria based on the $\mathbf{P}$ wave. Saunders $e t$ al. (1967) concluded that 'left atrial abnormality' was more accurate in describing $\mathbf{P}$ wave changes. In short, the use of electrocardiographic criteria for recognition of LA enlargement, although widely used, is unreliable.

Several comparisons have been reported of the various electrocardiogram criteria suggested for LA enlargement (Morris et al., 1964; Arevalo et al., 1963; Gooch et al., 1966; Saunders et al., 1967) and all indicate PTF-V $V_{1}$ to be the most sensitive. The present results indicated clearly that neither the duration nor the depth of the terminal portion of

TABLE 1. Results of PTF-V $V_{1}$ and LAD for seventy-four patients in three groups

\begin{tabular}{llrrrrr}
\hline & & \multicolumn{2}{c}{ PTF-V } & \multicolumn{2}{c}{ LAD } \\
\cline { 3 - 6 } & Group & No. & Mean & s.d. & Mean & s.d. \\
\hline 1. Normal subjects & 18 & $4 \cdot 1$ & $14 \cdot 2$ & $22 \cdot 0$ & $4 \cdot 2$ \\
2. Valvular disease & 36 & $39 \cdot 9$ & $31 \cdot 1$ & $35 \cdot 2$ & $10 \cdot 1$ \\
3. Hypertension and coronary artery disease & 20 & $18 \cdot 6$ & $24 \cdot 8$ & $28 \cdot 3$ & $7 \cdot 9$ \\
\hline
\end{tabular}

PTF- $V_{1}, P$ wave terminal force in lead $V_{1}$; LAD, left atrial dimension. 
the $\mathbf{P}$ wave was as sensitive an index as a combination of both.

The assumption that the size of the left atrium, an irregularly shaped chamber, is typified by a single antero-posterior dimension is supported by several correlative studies with left atrial cine-angiograms (Feigenbaum, 1972; Hirata et al., 1969; Ten Cate et al., 1974). The tendency of the chamber to assume a more spherical shape with enlargement lends further support to the validity of this assumption (Ten Cate et al., 1974). A large study reported of LA echocardiograms in normal subjects (Francis and Hagan, 1974) found the mean LAD to be $20.7 \mathrm{~mm}$ with s.d. $4.8 \mathrm{~mm}$. This is in close agreement with the normal values (group 1) in this series. Furthermore, the echo-derived measurement should not be affected by LA hypertrophy without enlargement, whereas PTF-V $V_{1}$ is (Kasser and Kennedy, 1969).

For these reasons it is suggested that the echocardiographic left atrial dimension is the best noninvasive test of left atrial size currently available. It should replace routine electrocardiography in the diagnosis, treatment, and follow-up of left atrial enlargement in cardiac patients.

\section{References}

Arevalo, A.C., Spagnuolo, M. \& Feinstein, A.R. (1963) A simple electrocardiographic indication of left atrial enlargement. Journal of the American Medical Association, $185,358$.

Berliner, K. \& MASTer, A.M. (1938) Mitral stenosis: correlation of electrocardiographic and pathological observations. Archives of Internal Medicine, 61, 39.

Chandraratna, P.A.N., Nanda, N.C. \& Shah, P.M. (1976) Echocardiographic study of the effects of acute left atrial hypertension on left atrial size. Journal of Clinical Ultrasound, 4, 15.

Chirife, R., Feitosa, G.S. \& Franki, W.S. (1975) Electrocardiographic detection of left atrial enlargement. Correlation of $\mathbf{P}$ wave with left atrial dimension by echocardiography. British Heart Journal, 37, 1281.

Feigenbaum, H. (1972) Echocardiography, 1st edn, pp. 152154. Lea and Febiger, Philadelphia.

Francis, G.S. \& HAGAN, A.D. (1974) Echocardiographic criteria of normal left atrial size in adults. Circulation, 49/50 (Suppl. III), 76.
Gooch, A.S., Celataud, J.B., Gorman, P.A., Saunders, J.L. \& CACERES, C.A. (1966) Leftward shift of the terminal P force in the ECG associated with left atrial enlargement. American Heart Journal, 71, 727.

Hirata, T., Wolfe, S.B., Popp, R.L., Helman, C.M. \& Feigenbaum, H. (1969) Estimation of left atrial size using ultrasound. American Heart Journal, 78, 43.

Human, G.P. \& Snyman, H.W. (1963) The value of the Macruz Index in the diagnosis of atrial enlargement. Circulation, 27, 935.

Hurst, J.W., Wenger, N.K., Cabrera, E., Estes, E.H. \& Hellerstein, H.K. (1963) Electrocardiographic Interpretation, 1st edn, pp. 251-252. McGraw-Hill, New York.

Ishikawa, K., Kini, P.M. \& Pipberger, H.V. (1973) P wave analysis in 2464 orthogonal electrocardiograms from normal subjects and patients with atrial overload. Circulation, 48, 565.

KASSER, I. \& KenNedy, J.W. (1969) The relationship of increased left atrial volume and pressure to abnormal $P$ waves on the electrocardiogram. Circulation, 39, 339.

LEWIS, T. (1920) The Mechanism and Graphic Registration of the Heart Beat. 1st edn. Paul B. Hoeber Co., New York.

Macruz, R., Perloff, J.K. \& Case, R.B. (1958) A method for the electrocardiographic recognition of atrial enlargement. Circulation, 17, 882.

MorRIS, J.J., Dunlop, W.M., Thompson, H.K., McInTosh, H.D. \& Estes, E.H. (1966) P wave analysis in the electrocardiographic diagnosis of left ventricular hypertrophy (abst.). Circulation, 31/32 (Suppl. II), 154.

Morris, J.J., Estes, E.H., Whalen, R.E., Thompson, H.K. \& McIntosh, H.D. (1964) $P$ wave analysis in valvular heart disease. Circulation, 29, 242.

Pipberger, H.V. \& Tanenbaum, H.L. (1958) The P wave, $\mathbf{P}-\mathbf{R}$ interval and $\mathbf{Q}-\mathbf{T}$ ratio of the normal orthogonad electrocardiogram. Circulation, 18, 1175.

Romhilt, D.W., Bare, K.E., Conradi, S. \& ScotT, R.D (1972) Morphologic significance of left atrial involvemento American Heart Journal, 83, 332.

Saunders, J.L., Colatayud, J.B., Schulz, K.J., Maranhas, V., GoOCH, A.S. \& GoldberG, H. (1967) Evaluation of ECG criteria for $P$ wave abnormalities. American Heart Journal, 74, 757.

Soloff, L. \& Zatuchini, J. (1958) Relationship of the P wave to left atrial volume in rheumatic heart disease with mitral stenosis. American Journal of Medical Science, 235, 290.

Ten Cate, F.J., Kloster, F.E., van Dorp, W.G., Meester, G.T. \& RoelandT, J. (1974) Dimensions and volumes of left atrium and ventricle determined by single beam echocardiography. British Heart Journal, 36, 737.

TeRMini, B.A. \& YU-CHEN LeE (1975) Echocardiographic and electrocardiographic criteria for diagnosing left atrial enlargement. Southern Medical Journal, 68, 161. 